\title{
Optogenetic activation of non-nociceptive $A \beta$ fibers induces neuropathic pain- like sensory and emotional behaviors after nerve injury in rats
}

\author{
Ryoichi Tashima ${ }^{1}$, Keisuke Koga', Misuzu Sekine, Kensho Kanehisa ${ }^{1}$, Yuta Kohro', \\ Yugo Fukazawa ${ }^{2}$, Kazuhide Inoue ${ }^{3}$, Hiromu Yawo ${ }^{4}$, Hidemasa Furue ${ }^{5}$, Makoto Tsuda ${ }^{1}$
}

${ }^{I}$ Department of Life Innovation, Graduate School of Pharmaceutical Sciences, Kyushu University, Japan, ${ }^{2}$ Department of Brain Structure and Function, Research Center for Child Mental Development, Faculty of Medical Sciences, Fukui University, Japan, ${ }^{3}$ Department of Molecular and System Pharmacology, Graduate School of Pharmaceutical Sciences, Kyushu University, Japan, ${ }^{4}$ Department of Developmental Biology and Neuroscience, Graduate School of Life Sciences, Tohoku University, Japan, ${ }^{5}$ Division of Neural Signaling, National Institute for Physiological Sciences, Japan

Neuropathic pain is caused by peripheral nerve injury (PNI). One hallmark symptom is allodynia (pain caused by normally innocuous stimuli), but its mechanistic underpinning remains elusive. Notably, whether selective stimulation of non-nociceptive primary afferent $\mathrm{A} \beta$ fibers indeed evokes neuropathic pain-like sensory and emotional behaviors after PNI is unknown, because of the lack of tools to manipulate $\mathrm{A} \beta$ fiber function in awake, freely moving animals. In this study, we used a transgenic rat line that enables stimulation of non-nociceptive $\mathrm{A} \beta$ fibers by a light-activated channel (channelrhodopsin-2). We found that illuminating light to the plantar skin of these rats with PNI elicited pain-like withdrawal behaviors that were resistant to morphine. Light illumination to the skin of PNI rats increased the number of spinal dorsal horn lamina I neurons positive to activity markers (c-Fos and phosphorylated extracellular signal-regulated protein kinase). Whole-cell recording revealed that optogenetic A $\beta$ fiber stimulation after PNI caused excitation of lamina I neurons, which were normally silent by this stimulation. Moreover, illuminating the hindpaw of PNI rats resulted in activation of central amygdaloid neurons and produced an aversion to illumination. Thus, these findings provide the first evidence that optogenetic activation of primary afferent $\mathrm{A} \beta$ fibers in PNI rats produces excitation of lamina I neurons and neuropathic pain-like behaviors that were resistant to morphine treatment. This approach may provide a new path for investigating circuits and behaviors of $\mathrm{A} \beta$ fiber-mediated neuropathic allodynia with sensory and emotional aspects after PNI and for discovering novel drugs to treat neuropathic pain. 